\title{
PEDAGOGÍAS DE LA ESPERANZA PARA LA EDUCACIÓN DEL NUEVO MILENIO
}

\section{MODIFICABILIDAD ESTRUCTURAL Y COGNIIIUA EDUCACIÓN EXPERIENCIAL}

German Pilonieta P.

“...La pedagogía como sinfonía, quedó reducida a una simple canción repetitiva, cantada por una pareja desgreñada, acompañada por un viejo piano desafinado".

"El desdibujamiento surgido en el perfil del maestro, ha hecho que solamente haya algunos, muy pocos músicos brillantes, que puedan atreverse a interpretar las nuevas partituras de la gran sinfonía de la pedagogía alternativa y liberadora".

Pilonieta 94.

\section{RESUMEN}

El artículo presenta a los docentes y demás profesionales de la educación, herramientas no tradicionales para el trabajo más importante del ejercicio docente que es la de generar condiciones propicias para aprender de manera contextualizada. Para ello, presenta un panorama histórico de la teoría de la modificabilidad estructural cognitiva con el fin de comprender su sentido y su poder en la educación del nuevo milenio. El artículo se inscribe en el marco de la democratización a través de las pedagogías de la esperanza. Se hace referencia a algunos de sus fundamentos conceptuales, operacionales e instrumentales y a las aplicaciones que en el mundo y en Colombia se vienen haciendo en el Equipo Cisne de Investigación y Desarrollo Pedagógico. Finaliza el artículo con una exposición sobre la educación experiencial, como otro enfoque universalmente válido para el desarrollo de competencias en todo terreno, especialmente en el campo de las organizaciones que aprenden y mucho más para las que producen, las cuales requieren hoy especializción y ambientes propicios de trabajo en equipo.

\section{PALABRAS CLAVE}

Modificabilidad, Equipo Cisne, educación experiencial, espacios dinámicos de formación, currículos situacionales, privación cultural, Reuven Feuerstein, funciones cognitivas, operaciones mentales, equidad.

\section{ABSTRACT}

The article provides the teachers and other professional of education with non traditional tools for the most important work of the teaching exercise which is to generate suitable conditions to learn from the context. To reach this, it presents a historical outlook of the cognitive structural ability to modify in order to understand its sense and power in the new millennium education. The article falls within the democratization framework through hope pedagogies. It refers to some conceptual, operational and instrumental foundations and to the applications being performed in the world and Colombia by the Cisne Team of Research and Pedagogical Development. The article ends with an exposition about experimental education as another approach universally valid for the competences development in all fields, especially in the organizations field which learn and much more for those that produce, which require nowadays specialized competences and appropriate environment to teamwork. 


\section{PRESENTACIÓN}

Para iniciar, quiero hacer algunas consideraciones, que creo, muy importantes, para poder comprender el sentido que tiene la teoría de la modificabilidad estructural cognitiva y de la educación experiencial, como aportes significativos a la capacidad de aprender, como factor fundamental del desarrollo, personal y comunitario, y la reducción de la inequidad.

En primer lugar, me parece que es necesario recordar que en estos momentos estamos sufriendo las consecuencias de lo que dejamos de hacer y de lo que hicimos mal en el pasado. La gran cantidad de personas que se encuentran en estados de pobreza, desarraigo, vulnerabilidad y en desventaja, es lo suficientemente grande, como para repensar enfoques, revalidar prácticas y crear nuevas formas de concebir y hacer democracia a través de la pedagogía y la educación, para lograr los estados de equidad y desarrollo necesarios.

Me parece que frente a esta situación en donde, definitivamente están involucrados

\section{RESEÑA DE AUTOR}

German Pilonieta, maestro y filósofo, Magister en educación, investigación educativa y análisis curricular. Trainer en Modificabilidad Estructural Cognitiva, Israel. Master Practitioner en PNL, España. Miembro fundador del Equipo Cisne de Investigación. Ha sido director general de educación de adultos del Ministerio de Educación Nacional y jefe de educación de adultos de la Secretaría de Educación del Distrito, Bogotá. Asesor de la Comisión V de la Cámara de Representantes. Ex director general de la Escuela Judicial del Ministerio de Justicia. Consultor del Departamento de Justicia de los Estados Unidos. Docente del Diplomado del Programa de Naciones Unidas para la fiscalización internacional de la droga. Docente en la maestría de currículo de la Universidad Javeriana. Docente del diplomado en Docencia Universitaria del Politécnico Grancolombiano. Asesor Internacional de Educación. Formador de Formadores. Medalla Simón Bolívar, 1994. Miembro fundador y correspondiente de la Academia Colombiana de Pedagogía y Educación. Miembro del comité científico de la revista internacional Magisterio. miles de niños y jóvenes pobres, y ricos, los verdaderos pedagogos tienen y deben decirle muchas cosas inteligentes y pertinentes a la educación.

Nuevos enfoques, nuevas miradas, nuevos abordajes son los que se requieren en esta sociedad del conocimiento y esa es precisamente la nueva tarea de la pedagogía. Lograr mucha mayor pertinencia de lo educativo en relación con nuevas dinámicas de desarrollo humano y el logro de la equidad.

Si bien es cierto que no es nada fácil llegar a comprender el problema, y mucho menos sus soluciones, esto no debe impedir que decididamente se busquen alternativas valiosas y pertinentes, para lograr mejores aproximaciones a un fenómeno tan complicado como es lo educativo, en relación con el desarrollo humano, en el marco de un estado de crisis generalizado.

Desde esta perspectiva es necesario comprender la teoría de la modificabilidad estructural cognitiva ya que su origen y nacimiento tuvo que ver con variables verdaderamente significativas que le dieron sentido, no sólo en el ámbito de la pedagogía, de la psicología y las tendencias cognitivas, sino desde la perspectiva de la democratización de la oportunidad.

En segundo término, creo necesario aclarar desde ahora que si bien es cierto que la razón que dio origen a la teoría de la modificabilidad fue muy particular, su validez es universal, sobre todo en situaciones en las que el síndrome de la privación cultural es evidente, causa desventaja y marginalidad.

En tercer lugar, debo advertir que esto es tan solo una aproximación a la teoría de la modificabilidad estructural cognitiva, de tipo informativo y que para su comprensión y aplicación se requiere, necesaria- 
mente, una entrega personal, profesional y una actitud de apertura comprometida en la acción formativa y mediadora.

Por último, confío poder compartir con fidelidad y autenticidad aquello que por voluntad propia he recibido, experimentado y construido en el seno del Equipo Cisne de Investigación, y que tiene fundamento no sólo en la teoría de la modificabilidad estructural cognitiva sino en la educación experiencial, como una contribución efectiva y pertinente a la consecución de la equidad y la construcción de la paz.

\section{INTRODUCCIÓN}

La modificabilidad estructural cognitiva, surge de las cenizas y las esperanzas de un pueblo, ocasionadas por una de las locuras más aberrantes que han creado los seres humanos sobre este planeta. La locura nazi.

El problema de la muerte en los campos de concentración, perfectamente diseñados para la eficiencia y la eficacia de logro del holocausto final, no puede dejar a la humanidad sino la enseñanza y la demostración del poder que los hombres podemos adquirir y desarrollar para la consecución de propósitos de diversa índole como los de Hitler. Esto no significa que hayan desaparecido más propósitos de esta misma naturaleza y hoy lo estamos apreciando cada momento. El equilibrio del terror.

La vida que renace, desde estos campos de concentración cuando sus puertas se abren por la llegada de los Aliados, en el ambiente más desolador que pueda encontrarse, es al mismo tiempo el momento del avivamiento de la llama de la poca espe- ranza que siempre se guardó como un secreto y con gran celo durante los incontables años de martirio y muerte, por parte de los sobrevivientes del holocausto.

El trabajo de la reconstrucción de la dignidad humana y todos los efectos secundarios convertidos posteriormente en preguntas, fue lo que dio comienzo a la reconstrucción de una esperanza que aún no termina y esto precisamente sustenta con una gran fuerza lo que muchos estamos haciendo en el terreno del amor convertido en pedagogía. Encontrar el cómo aprovechar las oportunidades, para democratizarlas y dirigir la acción pedagógica y educativa, en función de la formación de interlocutores válidos del desarrollo y constructores de la paz y la Nación.

Después de la guerra, bajo los auspicios de una organización juvenil israelí, Reuven Feuerstein, un judío rumano, discípulo de Piaget, que muy pronto descubrió su proyecto de vida, fue encargado de la integración, o mejor, de los procesos de reinserción de los niños y los jóvenes que sufrieron el proceso de deshumanización por la acción nazi y que ahora, libres, tenían que contribuir a la construcción de un pueblo y a la recuperación de la confianza en la naturaleza humana. Estamos hablando entonces de la verdadera "resiliencia" ${ }^{\star}$, es decir, de la forma como se realiza esta confianza a través de intervenciones, no sólo inteligentes, sino pertinentes con las estructuras cognitivas-afectivas de cada quien.

La diáspora judía, diseminada por todo el mundo, con multiplicidad de influencias, pero con la unidad de la religión y la fe, era poseedora de una serie de efectos

\footnotetext{
* Resiliencia: capacidad de una persona o grupo para seguir proyectándose en el futuro a pesar del acontecimientos desestabilizadores, de condiciones de vida difíciles y de traumas a veces graves. Tomado de: http:// www.psicologia-positiva.com/resiliencia.html
} 
tan negativos y tan complicados que hubo necesidad de replanteamientos y nuevas preguntas que pacientemente condujeron a formulaciones y reconceptualizaciones, no solo teóricas, sino liberadoras. Una de ellas, fue la del "síndrome de deprivación cultural”, el cual encierra un sin número de realidades y conceptos que hacen que la reflexión pedagógica y la acción educativa adquieran un sentido que va mucho más allá de lo escolar, como es el de la esperanza, la historia y la política.

Las aproximaciones y construcciones conceptuales que se han realizado y que dieron origen a la teoría de la modificabilidad estructural cognitiva y sus programas, surgieron de una realidad sufrida y encarnada por miles de personas y se extiende en cuanto manifestaciones y causas, a muchos países del mundo, como es el caso colombiano inscrito en nuestros niños, niñas y jóvenes pobres en donde hay que, definitivamente, aprender a formar seres "resilientes".

Muy pronto, la sistematicidad de estos trabajos fueron dando forma a los escritos que hoy conforman el patrimonio científico, no sólo del Instituto Internacional de Modificabilidad Cognitiva (El Hadassah, Wizo, Canada - Research) de Jerusalem sino de todos los que, de una manera u otra, tenemos que ver con este tipo de desarrollos en todo el mundo. Desarrollos relacionados necesariamente con las dimensiones del nuevo milenio y de los estados de crisis que nos están afectando de manera grave.

Tres son los programas iniciales que surgieron como respuestas pertinentes a las necesidades y a las preguntas que han orientado el trabajo del equipo de Feuerstein. El primero es el diagnóstico del potencial de aprendizaje (LPAD); el segundo, consiste en la "generación de experiencias positivas de aprendizaje mediado", Mediación (EAM); y el tercero es el de "Enriquecimiento instrumental” (PEI). Todos ellos son procesos que conllevan la formación personal y profesional que cualifica para su comprensión real y aplicación. También numerosas aplicaciones y derivados que vienen consolidándose en varios países del mundo, como el que el Equipo Cisne de Investigación realiza en Colombia.

\section{ALGUNOS CONCEPTOS FUNDAMENTALES}

El fundamento de la teoría de la modificabilidad estructural cognitiva se sustenta en un principio que se expresa de esta manera:

"El organismo humano es un sistema abierto, que en su evolución, adquirió la propensión para modificarse a sí mismo, siempre y cuando se genere un acto humano mediador".

Sobre este principio, que en su construcción conlleva muchas categorías conceptuales, algunas de ellas diferentes y hasta opuestas con las que la sicología tradicional ha defendido, se desarrolla todo el amplio panorama de modificabilidad. Representa un punto de partida un poco más pertinente y cercano que las concepciones comunes sobre la naturaleza del organismo humano y sobre todo con las concepciones de las estructuras cognitivas y afectivas que originan los comportamientos humanos.

Los constructos sobre la naturaleza de la inteligencia de tipo fijo e inamovible que derivó en prácticas educativas como las tradicionales, (además de estudios, investigaciones, métodos y medidas), son superados por la teoría de la modificabilidad, que desde su principio sustenta y concep- 
túa el organismo humano como poseedor de gran plasticidad y flexibilidad, que le permite efectivamente modificarse estructuralmente a sí mismo y desarrollarse de manera impredecible.

La modificabilidad define la inteligencia como la propensión o tendencia del organismo a ser transformado estructuralmente, como una forma de adaptación inteligente y sinérgica a nuevos estímulos, ya sean internos o externos. Modificabilidad conlleva un concepto dinámico en el desarrollo de la inteligencia, del aprendizaje y demás factores humanos.

Los comportamientos humanos, mirados e interpretados desde la modificabilidad, se conciben como generados por estados del organismo, como respuesta a los diversos estímulos, a diferencia de otros conceptos comunes como rasgos de inteligencia inamovibles.

Modificabilidad hace parte de las ciencias del comportamiento y del ámbito cognitivo, y es una opción para producir nuevos estados (no existentes) en las personas, así como nuevos modos de existencia, acciones y comportamientos. La razón de que modificabilidad tenga el poder de eliminar el síndrome de la deprivación cultural, factor perverso del desarrollo personal, hace que definitivamente se ubique en un lugar privilegiado en el marco del desarrollo pedagógico, educativo y comunitario. Va mucho más allá de las concepciones escolarizantes, para darle nuevos sentidos a los procesos de formación que deben coincidir con las fuerzas y tendencias del nuevo milenio. Las opciones, accesibles a todos los organismos humanos, independientemente de la edad, la etiología, y las condiciones específicas, (barreras infranqueables en el sistema edu- cativo tradicional), son consideradas como factores perfectamente aceptables y manejables para el desarrollo y el aprendizaje.

Factores negativos tales como los que puede traer la herencia, las condiciones genéticas, las anomalías cromosómicas, la edad extraescolar, los ambientes negativos, etc., difícilmente manejados por lo tradicional educativo, y que influyen poderosamente en los bloqueos, pueden ser superados por la posibilidad de la modificabilidad al ofrecer ciertas condiciones como la generación de experiencias positivas y sinérgicas de aprendizaje mediado. Se trata de una interacción altamente cualificada que definitivamente cambia la orientación del desarrollo cognitivo y afectivo de modo significativo.

Desde otra perspectiva, fuera de lo mencionado, la modificabilidad contempla fundamentalmente el trabajo intencional y especializado sobre las funciones cognitivas y las operaciones mentales. Cada uno de los programas trabaja de una manera u otra, funciones y operaciones, ya sea para realizar el diagnóstico del potencial de aprendizaje (LPAD), para aumentarlo y modificarlo (PEI) (o el programa de desarrollo de la capacidad para aprender y del pensamiento divergente del Equipo Cisne), por medio de la mediación (EAM).

Lo que se busca es la identificación del potencial intelectual y de aprendizaje de las personas para ejercer sobre estos, interacciones positivas y sinérgicas de modo que la inteligencia se enriquezca en los procesos y quede dispuesta para seguir creciendo y aprendiendo, a través de la interacción productiva, con los avances de la cultura, tanto nacional como internacional. Esto significa que va en dirección a fortalecer el fin último de la educación que es la autonomía.
La

modificabilidad define la inteligencia como la propensión o tendencia del organismo a ser transformado estructuralmente, como una forma de adaptación inteligente y sinérgica a nuevos estímulos, ya sean internoso externos. 
La calidad de la interacción que se realiza en la modificabilidad, afecta no sólo la estructura cognitiva, sino la emocionaly afectiva de las personas involucradas.
Modificabilidad y sus programas influyen en lo educativo de manera muy especial, cualificando especialmente la comprensión nueva de la persona y sus características, como la inteligencia, que es considerada como un proceso dinámico autorregulatorio que responde a la intervención ambiental externa.

La modificabilidad estructural cognitiva está caracterizada por tres criterios importantes que la hacen poseedora de un alto grado de permanencia, penetrabilidad y significado, como:

1. La relación entre la parte y el todo: en la transformación estructural existe una interdependencia en cuanto que al realizar cambios en las partes, estas afectan necesariamente el todo.

2. La transformación (de tipo estructural): ocurre en los procesos de cambio por medio de una gran variedad de condiciones, situaciones, modalidades y dominios gracias al funcionamiento de las operaciones mentales.

3. Continuación y auto perpetuación: en el sentido de que la modificabilidad se produce a lo largo de la vida de una persona y como fuerza sinérgica, ésta se aumenta en la medida en que se la utilice.

Se plantean además algunas modalidades del desarrollo cognitivo, que tienen que ver con el desarrollo cognitivo diferencial de las personas. Son dos: la exposición directa del organismo a los estímulos y la generación de experiencias positivas y sinérgicas de aprendizaje mediado.

La mediación (EAM) en su doble papel, el explicativo y el heurístico (en cuanto afirma la universalidad de la modificabilidad humana y el rol que desempeña en cuanto a la calidad de la intervención, en función de la modificabilidad estructural), hace que sea la llave maestra de todo el proceso, puesto que es el factor para el desarrollo de las funciones cognitivas más elevadas y la transformación cognitiva.

La generación de experiencias positivas y sinérgicas de aprendizaje mediado es posible, sólo si quien lo realiza, ha sido mediado, comprende y vive los doce criterios de mediación, y dirige su acción a la modificabilidad estructural, generando la propensión para que la persona pueda hacerla siempre en sí y por sí misma. La mediación es el acto de interacción que produce en la existencia de una persona, la flexibilidad, la auto plasticidad y le da la opción de ejercer la modificabilidad permanentemente.

La calidad de la interacción que se realiza en la modificabilidad, afecta no sólo la estructura cognitiva, sino la emocional y afectiva de las personas involucradas. Es una acción muy poderosa cuya definición operacional, permite, no sólo la comprensión de la modificabilidad, sino la generación de la predisposición para ella. El criterio de intencionalidad, es modificador para las dos personas que intervienen, y produce el criterio de reciprocidad, así como los demás criterios de mediación que se dan en ambas direcciones.

El resultado de este acto mediador consiste en la corrección de las funciones cognitivas, base de las operaciones mentales. Se trata de que el organismo humano fortalezca sus estructuras cognitivas y funcione de manera adecuada.

...cuanto mayor sea la experiencia de aprendizaje mediado y más pronto se someta la persona a dicha experiencia, mayor será la 
capacidad del organismo para ser modificado, además podrá utilizar eficazmente la estimulación directa a la que está sometido todo el organismo; por el contrario, cuanto menor sea la experiencia de aprendizaje mediado que se ofrezca a la persona, tanto cualitativa como cuantitativamente, menor será la capacidad del organismo para ser modificado y para utilizar la estimulación. (Feuerstein, 1973 y 1975).

Con respecto al manejo de los determinantes distales y proximales del desarrollo cognitivo diferencial, Modificabilidad y sus programas, tienen respuestas adecuadas, largamente probadas, que hacen de este enfoque de desarrollo, uno de los más pertinentes y poderosos en el terreno de la eliminación real de la privación cultural, la marginalidad y la desventaja social.

Desde la perspectiva pedagógica se puede decir que Modificabilidad, no admite cambios ni condiciones irreversibles, pues estos se pueden paliar o eliminar. No cree en la clasificación de las personas y rechaza la creencia de que el deterioro o daño genético y orgánico sean irreparables (sólo en casos extremos, en donde el juicio queda en suspenso hasta tanto se compruebe lo extremo del caso).

\section{APLICACIONES}

En el campo de la aplicación y desarrollo de la teoría de la modificabilidad estructural cognitiva se puede decir que durante estos años, desde que se dio a conocer en el mundo, las decisiones de su realización, han estado siempre ligadas, no al conocimiento de su existencia, sino al compromiso efectivo de personas que hemos comprendido, no sólo su sentido más auténtico, sino su profundidad y su per- tinencia en el camino de ser sembradores eficaces de esperanzas, en un contexto de desesperanza y de necesidad de desarrollo democrático.

Israel ha permitido la incursión directa de la modificabilidad en varios de sus programas educativos actuales. También lo ha hecho en la preparación y formación de profesionales en la seguridad aeronáutica y defensa del Estado. Gran Bretaña tomó la decisión de que toda la educación básica fuera orientada por los principios de modificabilidad. Francia, por su parte, ha avanzado mucho en la educación de adultos sobre estos principios. Mientras en Estados Unidos se están desarrollando con mucho éxito programas en varios estados y con varias instituciones. Asimismo, España con el Instituto Superior San Pío X en Madrid, ha logrado un gran avance en la aplicación y en la divulgación de las obras en castellano de modificabilidad para los países de América Latina.

Varios países de África, a pesar de sus problemas políticos, han introducido los programas de modificabilidad en el marco del desarrollo pedagógico.

En América Latina desde hace ya muchos años se vienen realizando, no sólo aplicaciones de sus programas, sino investigaciones y adaptaciones pertinentes para el trabajo con grupos específicos de personas. Son significativos los desarrollos en México, Guatemala, Argentina, Venezuela y Chile; en Colombia, el Equipo Cisne de Investigación, con sus programas de formación desarrollados con varios cientos de maestros de educación básica y media, con docentes universitarios y con profesionales de varias disciplinas, en los campos de la investigación y la docencia universitaria.
Gran Bretaña tomó la decisión de que toda la educación básica fuera orientada por los principios de modificabilidad. Francia, por su parte, ha avanzado mucho en la educación de adultos sobre estos principios. 
Recientemente se viene haciendo una fusión con aprendizaje experiencial lo cual ha resultado muy productivo $y$, sobre todo, significativo en el campo de la pedagogía y el desarrollo de nuevas habilidades y competencias.

\section{EDUCACIÓN EXPERIENCIAL}

La necesidad de consolidar la cultura de constructores de nación, hoy más que nunca es apremiante y la pedagogía y la educación deben comprometerse con esa misión $y$ ello nos debe obligar a replantear lo que hasta ahora hemos hecho.
Junto al desarrollo de modificabilidad, un poco más tarde, apareció un enfoque que nos ha parecido muy interesante y valioso. Se trata de la educación experiencial. También surgió de la mente de un judío alemán, Kurt Hanh, quien aportó experiencias significativas en las áreas de la convivencia pacífica y productiva.

Una de sus contribuciones más grandes al proyecto fue el planteamiento de los siete principios básicos, a saber:

1. Darle a los niños las oportunidades para el auto descubrimiento.

2. Hacer que los niños experimenten tanto el triunfo como la derrota.

3. Darles a los niños la oportunidad de ser eficaces en la causa común.

4. Generar períodos de silencio.

5. Entrenar la imaginación, la habilidad de anticipar y planear.

6. Hacer que los juegos sean importantes, pero no predominantes.

7. Liberar a los hijos de padres adinerados y con altas posiciones sociales, de la influencia paralizante de la riqueza y el privilegio.

El proyecto se extendió por todo el mundo, especialmente en Inglaterra y en los Estados Unidos en donde actualmente existen más de 10.000 campos especializados, como el que existe cerca de Bogotá, en el Parque Cisne.
El Equipo Cisne ha generado en estos años, procesos especializados, en donde involucra los principios de la mediación desde el enfoque de la modificabilidad estructural cognitiva y el programa de inteligencia y aprendizaje, en los desarrollos del aprendizaje experiencial, factor que no es manejado por ningún otro grupo que sólo hace ejercicios y simulaciones al aire libre o con notaciones de tipo out doors y otras denominaciones.

La esencia de este tipo de actividades especializadas tiene que ver con el desarrollo de las estructuras cognitivas a través de la exposición directa a retos y situaciones que exigen el desarrollo de la comunicación, el pensamiento estratégico, el trabajo en equipo, la confianza, el sentido de pertenencia, el manejo de logro y de fracaso, la sensibilidad al cambio, etc.

\section{FINALMENTE}

En esta ocasión, es importante que volteemos inteligentemente la mirada hacia aquellos que necesitan más de nosotros, no de lo que sabemos, sino de lo que hemos podido llegar a ser. Allí está la riqueza, en el mundo interior que hemos conformado y que crece cada vez, lo cual es la esencia del maestro.

Modificabilidad estructural y especialmente el programa de formación docente del equipo Cisne y todo lo que lo integra, ha facilitado ese camino de crecimiento interior, no sólo desde la comprensión de manejo de los procesos cognitivos, sino también desde la afectividad y la convivencia productiva en todos los niveles de la educación.

La necesidad de consolidar la cultura de constructores de nación, hoy más que 
nunca es apremiante y la pedagogía y la educación deben comprometerse con esa misión y ello nos debe obligar a replantear lo que hasta ahora hemos hecho.

El amor que nos llena, debe ser convertido en reflexiones pedagógicas y acciones educativas de gran significado, de tal manera que se supere lo escolarizante y se centre la atención en lo humano de las personas, en lo que nos hace verdaderamente felices y nos coloca en equidad y camino de hacer de este país algo que valga la pena.

Los invito a conocer y a involucrarse en el terreno de la modificabilidad estructural cognitiva y de la educación experiencial como oportunidad y como posibilidad, como forma de ser y hacer, como instrumento y apoyo de lo que estamos haciendo como complemento singular y como categoría conceptual que nos clarifica lo epistemológico y lo gnoseológico como punto de encuentro y de partida para diferenciar y hacer nuevas elaboraciones, en fin, como un espacio dinámico de formación en donde sean posibles los currículos estratégicos de tipo situacional.

Los años por venir, son definitivos y necesariamente debemos cambiar para poder hacer realidad los sueños de paz y libertad. No podemos esperar a que las cosas cambien, somos nosotros cuando cambiamos los que hacemos y, de esta forma, las cosas empiezan a transformarse. Llegó el tiempo de hacerlo y aquí estamos para apoyarlos en esto que es tan importante para su vida y para la de muchos otros.

\section{BIBLIOGRAFÍA}

- DELORS, Jacques (1998). La educación encierra un tesoro. México: UNESCO.
- FEUERTEIN, Reuven, et al. (1997) ¿Es modificable la inteligencia? Madrid: Bruño.

- KOZULIN, Alex (1994). La psicología de Vigotski. Madrid: Alianza.

- MARTÍNEZ, José María (1994). La mediación en el proceso de aprendizaje. Madrid: Bruño.

- MARTíneZ, José María (1991). Metodología de la mediación en el PEI. Madrid: Bruño.

- MORAles, Mario (1995). Estudio del niño de aprendizaje lento bajo dos enfoques de evaluación: Tradicional $y$ dinámica. Santiago de Chile: Universidad Católica.

- NOT, Louis (1994). Las pedagogías del conocimiento. México: Fondo de Cultura Económica.

- Pilonieta, Germán (1988). El currículo pertinente. Bogotá: Unisur.

- PIlonieTA, Germán (1994). 16 manuales para educación de adultos. Aprendiendo a mediar. Bogotá: SED.

- Pilonieta, Germán (1994). Trabajando por una cultura de educación de adultos en Santafé de Bogotá. Bogotá: SED.

- PILONIETA, Germán (1995). Proyecto educativo para los menores infractores $y$ contraventores. Bogotá: MEN.

- PILONIETA, Germán (1995). Proyecto educativo para jóvenes y adultos trabajadores. Bogotá: SED.

- Pilonieta, Germán (1996). Desarrollo de la inteligencia y del pensamiento divergente. Bogotá: Mimeo.

- PRIETO, María Dolores (1989). Modificabilidad cognitiva y PEI. Madrid: Bruño. 